\title{
DETECCIÓN DE CAPAS DE DESECHOS CON MÉTODOS GEOELÉCTRICOS: CASO RELLENO SANITARIO RÍO AZUL, COSTA RICA
}

\author{
DETECTION OF WASTE'S LAYERS WITH GEOELECTRIC METHODS: CASE OF \\ RIO AZUL SANITARY LANDFILL, COSTA RICA
}

\author{
Jorge Suárez* \& Héctor Zúñiga
}
Maestría en Hidrogeología y Manejo del Recurso Hídrico, Sistema de Estudios de Posgrado, Universidad de Costa Rica. Apartado 143-2010, Zapote, San José, Costa Rica
*Autor de contacto: jsuarez@geohydrogeo.com

(Recibido: 14/05/2013 ; aceptado: 26/11/2013)

\begin{abstract}
The Río Azul Landfill received waste for 34 years from various parts of the Greater Metropolitan Area, and for three decades was operated with a poorly planned waste disposal. The purpose was to identify areas of greatest potential leachate storage, making 25 VES (Vertical Electrical Soundings), whose effective exploration depth was around 65 meters. The proposed geophysical model consists of four layers: the first corresponds to a coverage of compacted material $(20 \Omega \mathrm{m}-54 \Omega \mathrm{m})$, the second layer contains unsaturated waste $(11 \Omega \mathrm{m}-18 \Omega \mathrm{m})$, the third $(1 \Omega \mathrm{m}-5.4$ $\Omega \mathrm{m})$ is saturated waste with leachate and the fourth $(49 \Omega \mathrm{m}-360 \Omega \mathrm{m})$ the basement on which was installed the landfill. Therefore, the geoelectric allowed determining the thickness and distribution of covering materials, waste, saturated waste with leachates and depth of the basement's top.

Keywords: Río Azul, geoelectrical prospecting, geophysics, VES, leachate, waste, sanitary landfill.

RESUMEN: El Relleno Sanitario Río Azul, recibió por 34 años desechos de diversas zonas de la Gran Área Metropolitana, y por tres décadas fue operado con una disposición de residuos poco planificada. El objetivo del estudio es identificar las zonas con mayores potenciales de almacenamiento de lixiviados a través de 25 SEV's (Sondeos Eléctri$\cos$ Verticales), cuya profundidad de prospección efectiva fue alrededor de $65 \mathrm{~m}$. El modelo geofísico planteado consta de cuatro capas: la primera corresponde a una cobertura de material compactado ( $20 \Omega \mathrm{m}-54 \Omega \mathrm{m}$ ); la segunda capa contiene desechos no saturados $(11 \Omega \mathrm{m}-18 \Omega \mathrm{m})$; la tercera $(1 \Omega \mathrm{m}-5,4 \Omega \mathrm{m})$ es de desechos saturados con lixiviados y la cuarta $(49 \Omega \mathrm{m}-360 \Omega \mathrm{m})$ el basamento sobre el cual se instaló el relleno sanitario. Por tanto, la geoeléctrica permitió determinar los espesores y distribución de los materiales de cobertura, desechos, desechos saturados con lixiviados y la profundidad del techo del basamento.

Palabras clave: Río Azul, prospección geoeléctrica, geofísica, SEV, lixiviados, desechos, relleno sanitario.
\end{abstract}




\section{INTRODUCCIÓN}

El Relleno Sanitario Río Azul (en adelante RSRA), con un área total de $0,47 \mathrm{~km}^{2}$, se encuentra ubicado en el Gran Área Metropolitana (GAM) de Costa Rica, tal como se muestra en la figura 1; recibió desde 1973 hasta el 2007 (34 años) residuos domésticos, industriales, hospitalarios, orgánicos y tecnológicos, generados por 12 cantones de la GAM. El proceso de degradación y descomposición de estos residuos tiene como uno de sus productos la generación de lixiviados, los cuales se caracterizan por presentar altas concentraciones de sólidos totales disueltos (STD), sales y el arrastre de metales pesados (Rojas, 2007). Estos lixiviados pueden fluir por gravedad hasta alcanzar las aguas subterráneas, por lo que son considerados el gran contaminante que podría generar la operación de un relleno.

Hasta el año 2013, según la revisión bibliográfica realizada, en el relleno sanitario de Río Azul se han estudiado los lixiviados sub - superficiales (los cuales son depurados en una planta de tratamiento). Sin embargo hasta el momento, no se han realizado estudios que evidencien la presencia o ausencia de lixiviados en profundidad. Por tanto, se propuso dar respuesta a esta interrogante mediante la aplicación de métodos de prospección geofísica, específicamente mediante la aplicación de métodos eléctricos (geoeléctrica). El uso de estos métodos ha sido utilizado mundialmente en basureros o vertederos por varios autores como por ejemplo Pomposiello (2009) en Argentina, Belmonte et al. (2008) en Oaxaca (México), Mota et al. (2004) en Portugal, y Meju (2000) en Leicester (Inglaterra).

Los objetivos de la investigación, son determinar los espesores y distribución espacial de las capas de desecho, así como la profundidad en la que se ubicaría el contacto con el basamento rocoso. Con esto, se pretende identificar las zonas con mayores potenciales de almacenamiento de lixiviados.

Los resultados aquí aportados, son parte de la información generada con la ejecución de los siguientes proyectos:

- Proyecto de investigación 830 - B0 222 "Evaluación geológica del relleno sanitario

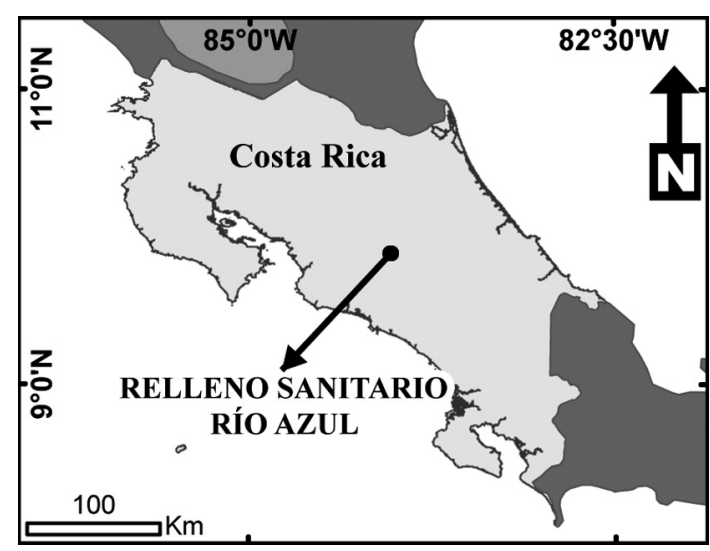

Fig. 1: Ubicación del área en estudio.

de Río Azul: etapa - post cierre", inscrito en la Vicerrectoría de Investigación de la Universidad de Costa Rica.

- Proyecto de tesis titulado "Evaluación Hidrogeológica del Relleno Sanitario de Río Azul" del Programa de Posgrado en Geología con Énfasis en Hidrogeología y Manejo del Recursos Hídrico de la Universidad de Costa Rica, el cual es desarrollado por el señor Jorge Suárez Matarrita, coautor de este trabajo.

\section{METODOLOGÍA}

Según Cantos (1987), los métodos geofísicos estudian la distribución lateral y en profundidad de propiedades físico - químicas de las capas del subsuelo, o de características relacionadas con dichas propiedades.

Arias (2007), indica que la geoeléctrica es uno de los métodos geofísicos de prospección con mayor difusión y aplicación en las ciencias de la Tierra. Consiste en la inyección de corriente eléctrica (I) al subsuelo y a partir del potencial $(\Delta \mathrm{V})$ generado en el mismo se determina la resistencia (R) del suelo. Este valor de resistencia es transformado a un valor de resistividad aparente $(\rho a)$, a partir de una constante geométrica (K) cuya magnitud dependerá del dispositivo empleado y de la separación de los electrodos, tanto de inyección de corriente (A, B) como de medición del potencial $(\mathrm{M}, \mathrm{N})$. 
El sondeo eléctrico vertical (SEV), utiliza cuatro electrodos geoeléctricos ( $\mathrm{A}, \mathrm{B}, \mathrm{M}, \mathrm{N})$, los cuales dependiendo de su ubicación y distancia dan origen a los diversos dispositivos existentes. En estos sondeos, el centro del dispositivo de los electrodos es fijo y el espaciamiento entre ellos va aumentando para obtener información a mayor profundidad. (Arias, 2007).

En esta investigación, se decidió aplicar este tipo de métodos, ya que con ellos se obtienen buenos resultados al definir capas subterráneas en medios con altos contrastes de conductividad eléctrica. Es de esperar que las capas de desechos presenten una concentración relativamente elevada de sales y sólidos totales disueltos, que favorecerá una alta conductividad del medio. En contraparte, se espera que la conductividad eléctrica sea más baja en las zonas con ausencia de desechos. En el sitio se realizaron un total de 25 SEV's aplicando el dispositivo Schlumberger, estando ubicados estos en sitios estratégicos para formular 3 perfiles de correlación según las resistividades obtenidas (Fig. 2).

El dispositivo Schlumberger (Fig. 3) consta de dos electrodos ( $\mathrm{M}$ y N) que miden la diferencia de potencial " $\Delta \mathrm{V}$ " (en milivoltios), los cuales, están ubicados en medio de otros dos electrodos (A y B) en los que se inyecta la corriente "I" (en miliamperios). La relación $\mathrm{AB} / \mathrm{MN}$ debe ser lo más grande posible, cumpliéndose que $\mathrm{AB}$ debe ser al menos de cuatro a veinte veces mayor que MN. (Arias, 2002).

La profundidad a la que se realiza el sondeo está en función de la separación $\mathrm{AB}$, del medio y de la corriente, por lo que el procedimiento habitual contempla desplazamientos simétricos de A y $\mathrm{B}$, los cuales han sido definidos previamente. La separación entre los electrodos $\mathrm{M}$ y N se mantiene constante, y solo debe aumentarse dicha separación cuando medir la diferencia de potencial se

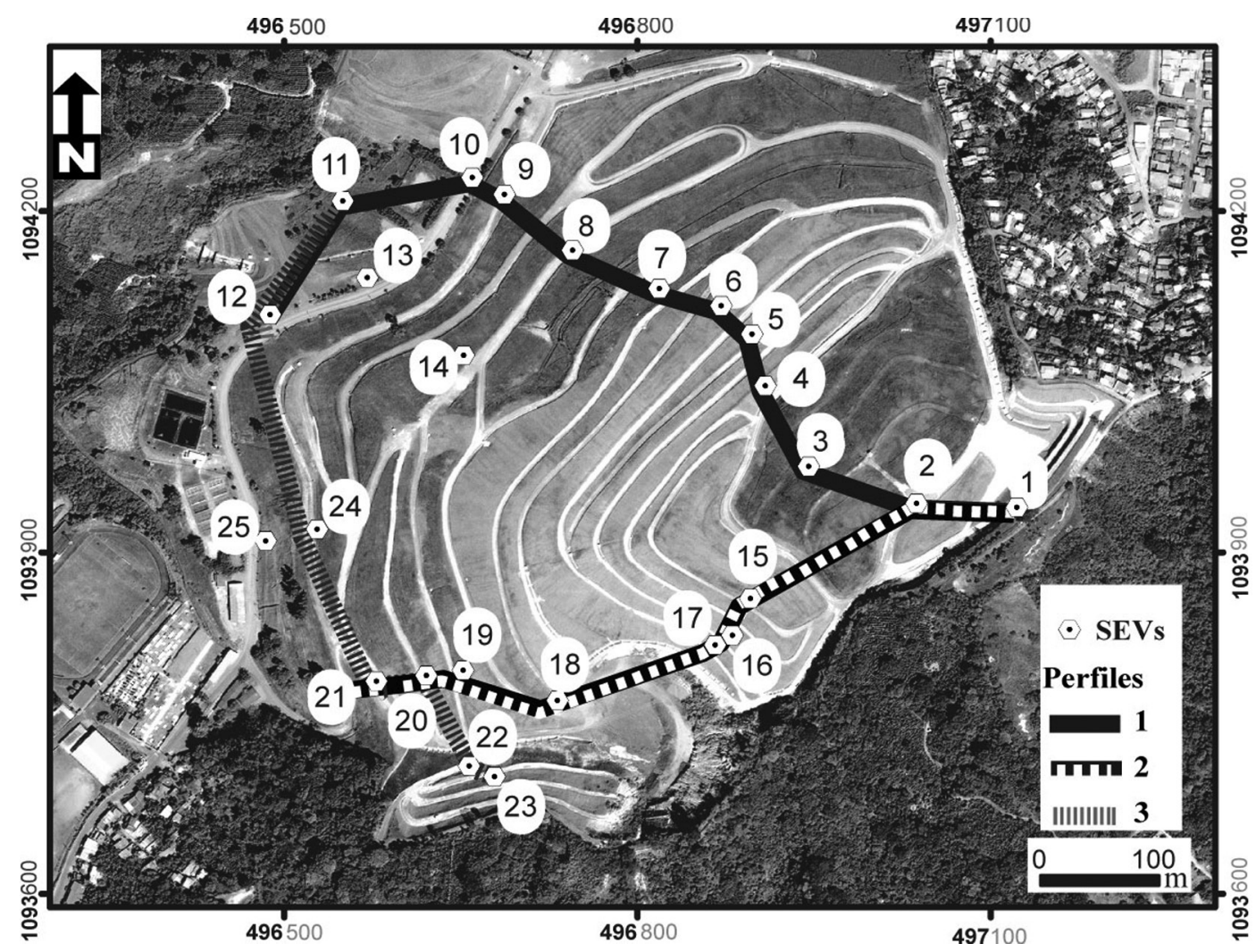

Fig. 2: Ubicación de sitios de prospección geofísica. 


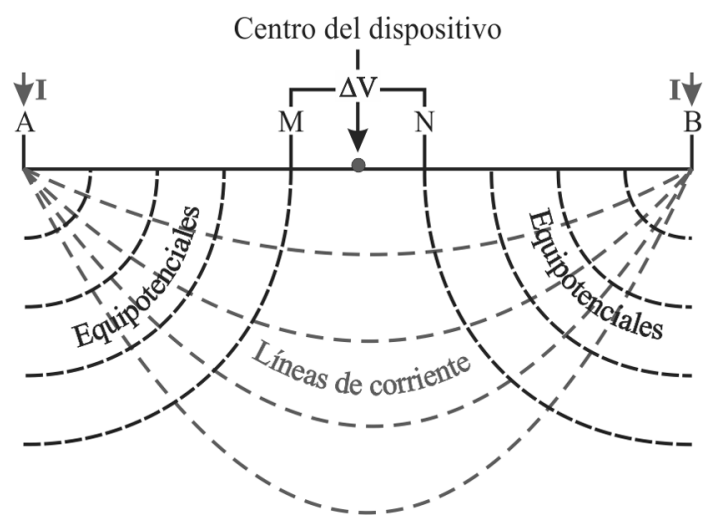

Fig. 3: Esquema general del dispositivo Schlumberger.

torna difícil. El aspecto más importante en este dispositivo es mantener un voltaje suficientemente grande para ser medido, ya que conforme aumenta la separación entre A y B, la diferencia de potencial disminuye. (Arias, 2002)

Para la ejecución de los SEV's en el campo, se utilizó el instrumento TERRAMETER SAS 1000 (propiedad de la Escuela Centroamericana de Geología de la Universidad de Costa Rica), junto con los accesorios respectivos para este tipo de ensayo. Dadas las limitaciones de espacio en el sitio, la mayor abertura $\mathrm{AB} / 2$ utilizada fue de $150 \mathrm{~m}$.

La inversión de los datos de campo del SEV se realizó con el programa de cómputo IP2WIN (versión 3.01, de Moscow State University), licencia libre.

\section{CONTEXTO GEOLÓGICO}

Suárez \& Malavassi (1953) indican que el cerro Asilo estaría constituido por dos tipos de litología. En el sector noroeste de este cerro mencionan la presencia de una unidad sedimentaria constituida por areniscas finas y margas; hacia la vertiente sureste del cerro, sobreyace la unidad volcánica constituida por porfiritas andesíticas y basaltos.

Según estos autores, las porfiritas andesíticas son de color gris claro y se pueden presentar ya sea en diques o como cuerpos masivos. La porfirita en masa es muy peculiar, pues puede confundirse con una andesita.
Franco (1977) indica que el cerro Asilo estaría constituido básicamente por coladas de lava, tobas, ignimbrita y tobas de lapilli. Estos materiales los agrupan en la Unidades Asilo y Río Azul, describiéndolas de la siguiente forma:

- Unidad Asilo: corresponde a la lava que aflora en el cerro Asilo. Presenta textura afanítica - porfirítica con un $25 \%$ de fenocristales xenomórficos de plagioclasa, $10 \%$ de cristales idiomórficos aciculares de anfíboles dispuestos en posición radial, $2 \%$ de fragmentos líticos de naturaleza volcánica variando de $3-12 \mathrm{~mm}$ de tamaño con forma sub - redondeada a angulosa $\mathrm{y}$ tonalidades grises verdosas. En el flanco oriental del cerro, es posible observar los diferentes grados de meteorización hasta culminar con una arenisca fina, blanca muy caolinitizada. El contacto inferior es totalmente discordante con las unidades y formaciones adyacentes. Su edad sería cuaternaria por constituir uno de los derrames lávicos procedentes del volcanismo intenso que dio origen a los cerros de la Carpintera.

- Unidad Río Azul: denomina así a las tobas compactas, ignimbritas y tobas de lapilli que afloran en el flanco occidental del cerro Asilo depositadas en ambiente lacustre. En la base se encuentra una toba muy compactada, blanca verdosa con cristales de plagioclasa alterados y óxidos de hierro y manganeso así como manchas obscuras de materia orgánica. Está intruida por dos diques muy alterados y friables cuya matriz varía de amarillo - verdoso obscuro a castaño - oliva claro con fenocristales de plagioclasa arcillificada; tienen un ancho de 0,5-0,6 m. Sobreyaciendo estas tobas, se encuentra un estrato de tobas de lapilli de $30 \mathrm{~cm}$ de espesor. Los fragmentos de naturaleza volcánica, son angulosos variando hasta $23 \mathrm{~mm}$ de tamaño incluidos dentro de una matriz arcillosa que también cementa abundante pómez; debido a la fuerte alteración, las coloraciones son variadas dominando el púrpura moderado y el rojo brillante. Seguidamente se sitúa un estrato formado por ignimbritas, cuya base es moderadamente compacta y muy alterada, mostrando sus fiammes y fragmentos líticos aplanados bien orientados en una dirección; los fragmentos son redondeados a sub - redondeados a redondeados lo que indica un transporte en un medio acuático. Las tonalidades 
son púrpura pálido y púrpura grisáceo, mientras que las fiammes son pardo - verdosas. El techo es menos compacto, fuertemente arcillificado color verde azulado; los fragmentos líticos angulosos varían de $2-15 \mathrm{~mm}$ y las fiammes de $2-5 \mathrm{~mm}$ de longitud en forma de media luna cuya coloración es más intensa que la matriz y su orientación regular. Exteriormente, la roca está cubierta con óxidos de hierro y dendritas de pirolusita. La secuencia culmina con una toba blanca hasta rosada moderada, muy friable y arcillificada con abundancia de óxidos. Su edad sería del Cuaternario.

Finalmente, Denyer \& Arias (1990) muestran que el cerro Asilo estaría conformado por materiales de la Formación Grifo Alto, la cual definen (Denyer \& Arias, 1991) como lavas andesíticas, algunas con augita y/o hipersteno de color gris a rojizo. Se encuentran algunos pocos basaltos y dacitas. Hay además flujos piroclásticos gruesos con bloques de lavas y escorias angulares y con tamaños decimétricos. Su edad sería no mayor a 4 m.a., teniendo así una edad Plioceno-Pleistoceno.

La Formación Grifo Alto sobreyace discordantemente la Formación La Cruz y la Formación Coris y es sobreyacida por las Lavas Intracañón y los Depósitos de Avalancha Ardiente.

Según observaciones de campo, se constató que el cerro Asilo está constituido mayoritariamente por lavas, generalmente de composición andesítica. Sin embargo, hacia el núcleo del cerro, las muestras observadas indican un alto contenido de fenocristales de hornblenda, lo que correspondería más a una composición dacítica. Así también, llama la atención el alto contenido de inclusiones de rocas ígneas, de composición igualmente dacítica, tamaño muy variado y el color de la matriz muestra una amplia variación entre tonos de grises.

Estos elementos corresponden con lo descrito por Franco (1977) para la Unidad Asilo y lo descrito por Denyer \& Arias (1991) para la Formación Grifo Alto.

No obstante, las modificaciones en el terreno, así como las toneladas de desechos depositadas en el sitio, hacen que lo descrito por Franco (1977) para Unidad Río Azul ya no sea fácil de observar en el campo. El único elemento descrito por este autor que fue posible identificar corresponde a la toba de lapilli, la cual se observa en la margen izquierda de la quebrada Azul, observándose fragmentos angulosos posiblemente de pómez, donde el color de la matriz era generalmente café - rojizo.

Por su naturaleza, este contexto geológico sugiere que estos materiales (rocas ígneas) deberían presentar resistividades relativamente altas con respecto a las que se esperarían registrar en el depósito de desechos. Sin embargo, los materiales que constituyen el basamento del RSRA, muestran un alto grado de meteorización, donde incluso los feldespatos generalmente ya se han transformado en arcillas, y por lo tanto, las resistividades esperadas ya no serán muy altas (de orden de los miles de $\Omega \mathrm{m}$ ), sino más bien moderadamente altas (centenas de $\Omega \mathrm{m}$ ).

\section{INTERPRETACIÓN DE LOS SONDEOS}

Para la interpretación de los SEV's, fue necesario digitar primero los datos de campo en hojas logarítmicas y se aplicó la respectiva corrección en los puntos de traslape, esto con el fin de obtener una única curva continua. La interpretación de las curvas de resistividad se realizó utilizando como herramienta el software libre IPI2Win para cada uno de los 25 SEV's. Con dicho software se determinaron los valores de las resistividades reales y los espesores de las diferentes capas, generando un modelo Geoléctrico para cada SEV.

Los criterios empleados para la interpretación de los datos fueron: contexto espacial, análisis cualitativo según Orellana (1972), las magnitudes relativas y el contexto geofísico. En general, los resultados obtenidos con la inversión de los datos fueron los siguientes:

\section{Sondeos para caracterizar la respuesta del basamento}

Con este fin se realizaron los SEV's 1, 22 y 23. El SEV1 es el más representativo de este grupo, y se realizó en la cima del cerro Asilo, en una zona donde la roca considerada el basamento no fue cubierta con desechos durante la operación del RSRA. Según se observó en el campo, esta roca 
sería una lava andesítica muy meteorizada. Esto se refleja en el rango de resistividades obtenidas, las cuales varían entre 111 y $205 \Omega \mathrm{m}$. Del análisis total de los SEV's de este grupo, y como se observa con claridad en la Figura 4 y en el Cuadro 1 , se define un basamento cuyas magnitudes de resistividad superan los $100 \Omega \mathrm{m}$.

Para el SEV1, la forma de la curva se cataloga como de tipo K, según el método cualitativo en Orellana (1972); por lo tanto, la segunda capa es la más resistiva entre extremos de menor resistividad eléctrica.

De las tres capas definidas, la primera (con un espesor aproximado de $0,5 \mathrm{~m}$ ) con una resistividad de $165 \Omega \mathrm{m}$, es interpretada como un suelo residual generado por la meteorización de la roca madre. La segunda capa presenta una resistividad de $205 \Omega \mathrm{m}$; se asocia con rocas ígneas relativamente menos meteorizadas, cuyo espesor es de $5 \mathrm{~m}$, hasta entrar en contacto con la última capa $(111 \Omega \mathrm{m})$ de la misma roca pero quizá con un posible aumento en el contenido de humedad, ya que la magnitud de la resistividad disminuyó, pero no significativamente.

\section{Modelo de tres capas}

En este grupo se incluyen los SEV's 10, 12, 14 y 15. El SEV10 (curva tipo: H) es representativo para el modelo de tres capas ubicado en los sitios con presencia de desechos (Figura 5, Cuadro 2). La primera capa corresponde a una cobertura, la cual es una calle de lastre con un espesor de $2,5 \mathrm{~m} \mathrm{y}$ $54 \Omega \mathrm{m}$ de resistividad eléctrica. La segunda posee

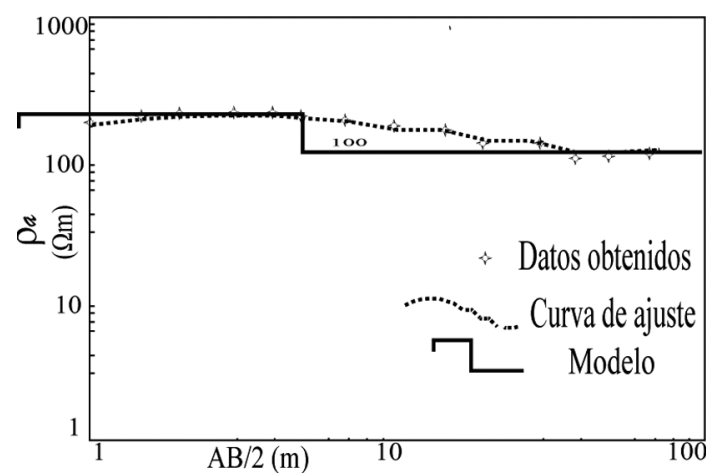

Fig. 4: SEV1, modelo de tres capas ubicado sobre el basamento.

resistividad de $4 \Omega \mathrm{m}$ y un espesor de $37 \mathrm{~m}$ correlacionado con desechos saturados de lixiviados. La tercera y última capa se considera el basamento debido a las altas resistividades eléctricas (300 $\Omega \mathrm{m})$ y se ubica a $39,5 \mathrm{~m}$ de profundidad.

En general, el error de ajuste fue de $4,8 \%$ en promedio en este grupo de sondeos.

\section{Modelo de cuatro capas}

De los 25 SEV's realizados, en 13 de ellos se obtuvo un modelo que sugiere la presencia de cuatro capas (SEV's 2, 5, 6, 7, 9, 10, 11, 13, 16, $18,20,21$ y 24$)$, donde el error promedio de ajuste fue de 5,13\%. El SEV5 es el más representativo de este grupo, tiene un tipo de curva $\mathrm{QH}$, la cual es compuesta por presentar por una disminución gradual de las resistividades hasta topar con un aumento en el último estrato. Las resistividades están en el rango desde los $4 \Omega \mathrm{m}$ hasta los $100 \Omega \mathrm{m}$.

Cuadro 1

Características de la inversión del SEV1 (Caracterización del basamento)

\begin{tabular}{ccccc}
\hline Capa N $^{\circ}$ & Resistividad real $(\Omega \mathrm{m})$ & Espesor $(\mathrm{m})$ & Profundidadal techo $(\mathrm{m})$ & Correlación \\
\hline 1 & 165 & 0,5 & 0,5 & Suelo residual \\
2 & 205 & 5,0 & 5,5 & Basamento de Lavas andesíticas \\
3 & 111 & - & - & Basamento Lavas (andesitas) con humedad \\
\hline
\end{tabular}




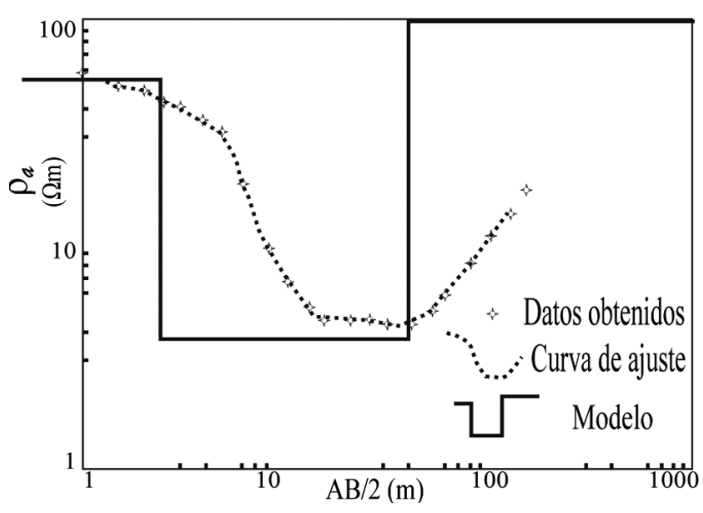

Fig. 5. SEV10, modelo de 3 capas ubicado sobre desechos.

Según se muestra en la figura $6 \mathrm{y}$ en el Cuadro 3, la primera capa (25 $\Omega \mathrm{m})$ se correlaciona con el suelo tipo cobertura, cuyo espesor es de 1,5 m. Esta capa es subyacida por lo que correspondería a $10 \mathrm{~m}$ de desechos, los cuales muestran una resistividad de $13 \Omega \mathrm{m}$. Bajo esta capa, se ubica un depósito de desechos posiblemente saturados de lixiviados, ya que presenta una resistividad muy baja (4 $\Omega \mathrm{m})$. Como capa final se encuentra el basamento ígneo, cuya resistividad real es de $100 \Omega \mathrm{m}$.

\section{Modelo de cinco capas}

En los SEV's 3, 4, 8 y 19 el modelo obtenido sugiere la presencia de cinco capas (con un error de ajuste de 5,6\%), siendo el SEV4 el más representativo de este grupo, el cual es de tipo HH. La ubicación de este sondeo se escogió porque se tiene certeza que en este punto hay desechos sepultados.

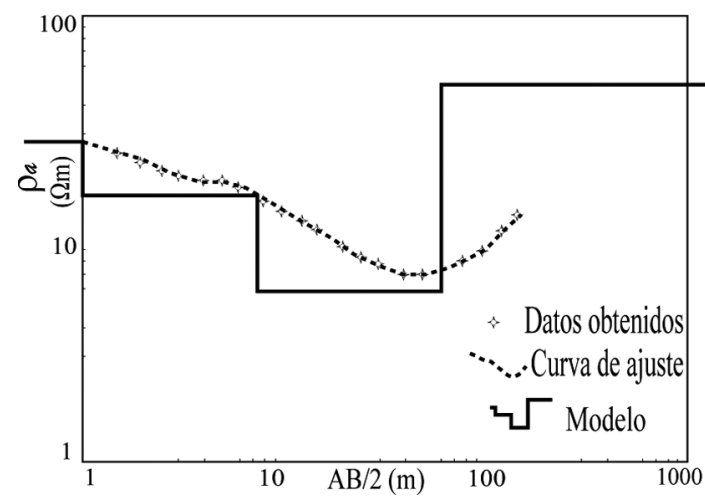

Fig. 6: SEV5, modelo de 4 capas.

Como se muestra en la figura $7 \mathrm{y}$ en el Cuadro 4 , el rango de las magnitudes de resistividad varió drásticamente desde $1,0 \Omega \mathrm{m}$ hasta $300 \Omega \mathrm{m}$ en las cinco capas detectadas. La primera de $0,9 \mathrm{~m}$ de espesor correspondería al suelo, que en este caso es una "cobertura" y presenta una resistividad de $20 \Omega \mathrm{m}$. La segunda capa de 2,2 $\mathrm{m}$ de espesor se considera que está constituida por desechos con presencia de lixiviados, ya que tiene un valor muy bajo de resistividad eléctrica $(6,0 \Omega \mathrm{m})$, lo cual sería una manifestación de la presencia de materiales conductores como sales, líquidos, entre otros, que permiten transmitir la electricidad con facilidad.

La tercera capa presenta un valor de $56 \Omega \mathrm{m}$ a una profundidad entre $\operatorname{los} 3,1 \mathrm{~m}$ hasta $\operatorname{los} 7,1 \mathrm{~m}$ (espesor de $4 \mathrm{~m}$ ), por lo que se correlaciona con un lente de cobertura antigua. Además, bajo él, se ubica otra capa de desechos saturada de lixiviados con valores de resistividad de $1,0 \Omega \mathrm{m}$.

Finalmente la quinta capa se correlaciona con el basamento de lavas ya que los valores

Cuadro 2

Características de la inversión del SEV10 (Modelo de tres capas)

\begin{tabular}{ccccc}
\hline Capa N & Resistividadreal $(\Omega \mathrm{m})$ & Espesor $(\mathrm{m})$ & Profundidad al techo $(\mathrm{m})$ & Correlación \\
\hline 1 & 54 & 2,5 & 2,5 & Cobertura tipo calle \\
2 & 4 & 37 & 39,5 & Basura saturada de lixiviados \\
3 & 300 & & & Basamento lávico \\
\hline
\end{tabular}




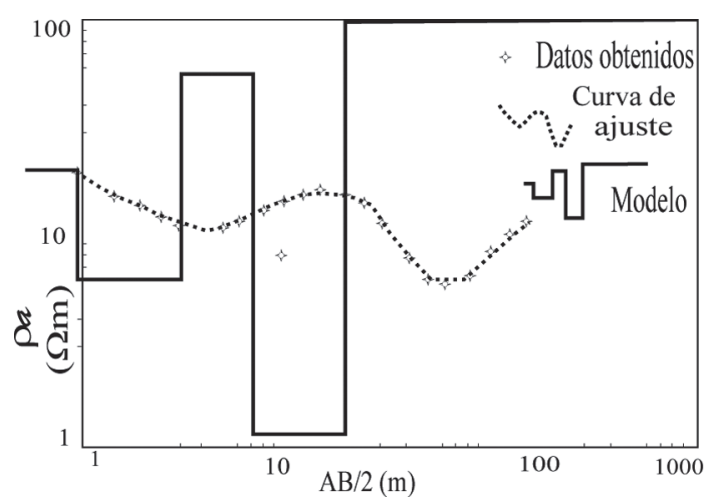

Fig. 7: SEV4, modelo de 5 capas. Presencia de desechos con lixiviados.

aumentan hasta los $300 \Omega \mathrm{m}$, lo cual es un contraste contundente y ejemplifica el contacto de los lixiviados con roca madre.

\section{ANÁLISIS DE PERFILES GEOELÉCTRICOS}

Con los datos de los SEV's, se realizaron perfiles geoeléctricos de correlación que permiten apreciar de mejor forma la distribución especial de los resultados obtenidos, así como la interpretación de los mismos.

\section{Perfil 1}

Como se observa en la figura 2, el Perfil 1 se elaboró utilizando los resultados obtenidos con los SEV's 1 al 12. En él se observa claramente el contraste vertical de la roca sana y el área intervenida (Figura 8).

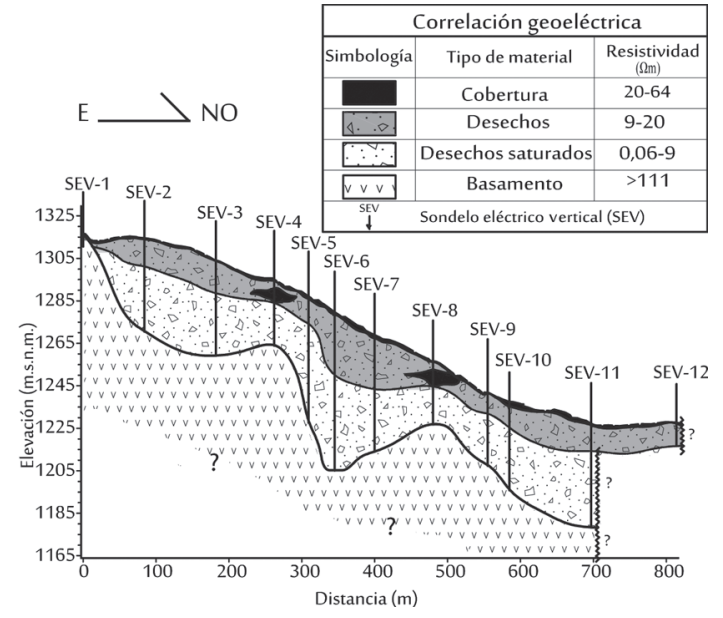

Fig. 8: Perfil geoeléctrico 1

A partir del SEV2 al SEV10 se presentan cuatro capas: una en superficie que corresponde al material de cobertura y calles en lastres, otra subyacente con desechos no saturados, la tercera de desechos con lixiviados, y la última que correspondería con el basamento de rocas volcánicas.

Se observa una interconexión espacial horizontal de las capas de desechos con lixiviados, lo que sugiere una pluma de contaminación subterránea que drena gradiente abajo y en parte descarga en la quebrada Río Azul, contaminando las aguas subterráneas, esto se constató en el proceso de realización de un estudio hidrogeológico.

Se pueden apreciar la morfología irregular del basamento, la cual se detecta como una zona de transición entre desechos con lixiviados (conductores) y el basamento (resistivo), marcado por el aumento en los valores de resistividad.

Cuadro 3

Características de la inversión del SEV5 (Modelo de 4 capas)

\begin{tabular}{ccccc}
\hline Capa $\mathrm{N}^{\mathrm{o}}$ & Resistividad real $(\Omega \mathrm{m})$ & Espesor $(\mathrm{m})$ & Profundidad al techo $(\mathrm{m})$ & Correlación \\
\hline 1 & 25 & 1,5 & 1,5 & Cobertura tipo suelo \\
2 & 13 & 10 & 11,5 & Desechos \\
3 & 4 & 35 & 46,5 & Desechos saturados de lixiviados \\
4 & 100 & - & - & Basamento lávico \\
\hline
\end{tabular}


También se puede apreciar la presencia de antiguos materiales de cobertura enterrados entre las capas de desechos, los cuales están distribuidos en forma lenticular y asistemáticamente en el perfil.

Explotar la forma de las celdas de desechos y la morfología del basamento en los perfiles 1 y 2 , indicando espesores máximos obtenidos a partir de la interpretación.

\section{Perfil 2}

Se plantea esta sección a partir de los SEV's 1 y 2, además de los SEV's 15 - 21. En él (Fig. 9) también se aprecia con claridad el contraste vertical del basamento y el área intervenida con depósitos de basura a lo largo de este perfil. Casi en la totalidad de la sección, son detectadas capas de desechos saturadas con lixiviados posiblemente. Estas capas muestran continuidad lateralmente, por lo que la pluma de contaminación de lixiviados se extendería gradiente abajo, hacia el SEV 21.

El límite de la zona saturada por lixiviados es fácilmente detectada entre los SEV's $2-15$ y del 19 - 21. La respuesta del basamento se manifestó con claridad a través de todo el perfil, caracterizado por resistividades altas mayores a $200 \Omega \mathrm{m}$.

$\mathrm{Al}$ igual que en la figura 8 , se pueden distinguir claramente las capas de cobertura, las cuales se pueden correlacionar principalmente con suelos o en algunos casos con el lastre compactado de las vías de acceso.

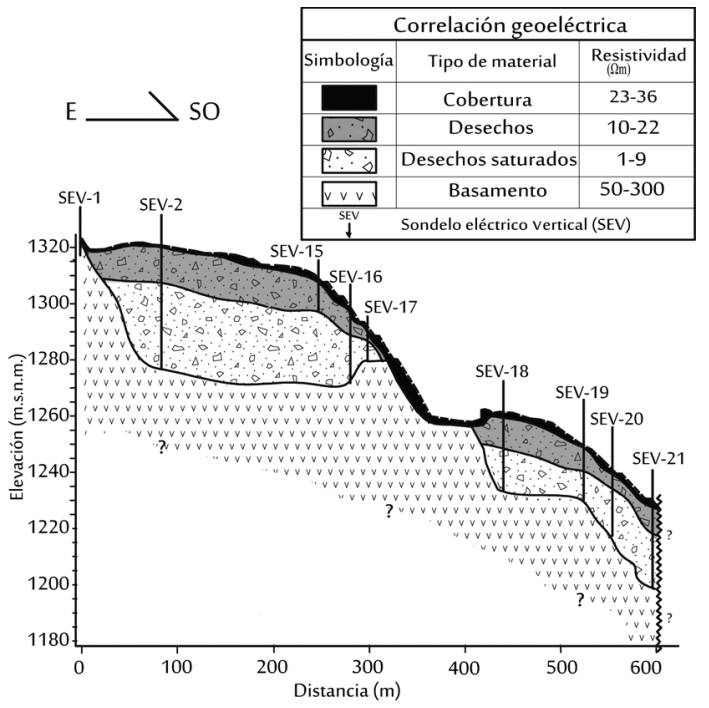

Fig. 9. Perfil geoeléctrico 2.

\section{Perfil 3}

En esta sección (Fig. 10), elaborado a partir de la correlación de los SEV's 23, 22, 19, 21, 24, 12 y 11 , se detecta el contraste vertical del basamento ígneo (SEV's 23 y 22) con el depósito de desechos (SEV's 21 - 11). El perfil permite apreciar la acumulación de desechos no saturados y desechos saturados. Estas capas sobreyacen al basamento que tiene resistividades superiores a los $50 \Omega \mathrm{m}$.

\section{CONCLUSIONES}

Los resultados obtenidos utilizando el método geoeléctrico fueron certeros en determinar el

Cuadro 4

Características de la inversión del SEV4 (Modelo de cinco capas)

\begin{tabular}{ccccc}
\hline Capa N $^{\text {o }}$ & Resistividad real $(\Omega \mathrm{m})$ & Espesor $(\mathrm{m})$ & Profundidad al techo $(\mathrm{m})$ & Correlación \\
\hline 1 & 20 & 0,9 & 0,9 & Cobertura tipo suelo \\
2 & 6 & 2,2 & 3,1 & Desechos saturados con lixiviados \\
3 & 56 & 4 & 7,1 & Cobertura antigua \\
4 & 1 & 13 & 20,1 & Desechos saturados de lixiviados \\
5 & 300 & & & Basamento de lavas \\
\hline
\end{tabular}




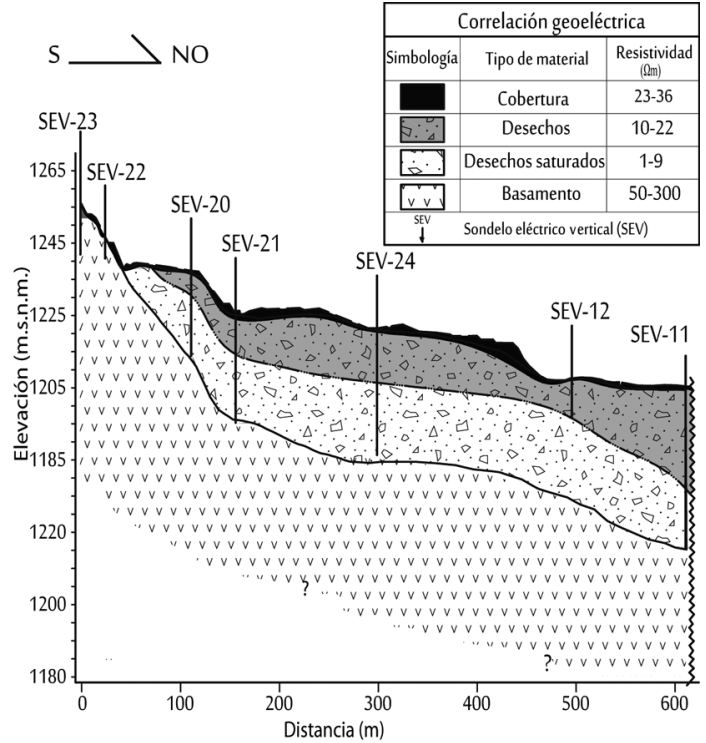

Fig. 10. Perfil geoeléctrico 3.

número de capas, su posible naturaleza y el espesor de las mismas.

Esto permitió plantear un modelo geoeléctrico general de cuatro capas, que aporta detalles acerca de la distribución geométrica del depósito de desechos en el Relleno Sanitario de Río Azul, aspecto del cual se contaba con poca información. A continuación una descripción de cada capa:

- Capa 1: presenta una resistividad que varía entre $20 \Omega \mathrm{m}$ y $54 \Omega \mathrm{m}$, relacionándose con la presencia de una cobertura de material compactado característico de un camino de lastre (grava con arcilla), así como de una cobertura tipo suelo compactado, utilizado como capa sello o de cobertura de los desechos depositados en el relleno sanitario. Los espesores varían entre 0,5 a 3,0 m.

- Capa 2: presenta una resistividad entre $11 \Omega \mathrm{m}$ y $18 \Omega \mathrm{m}$. Se correlaciona con la presencia de desechos depositados en el sitio, cuya concentración mayor de iones favorece el flujo de la corriente por el medio, y por tanto genera una disminución en las magnitudes de la resistividad. Su espesor varía entre 4,0 y 12,0 m, alcanzando su profundidad máxima en el SEV2.
- Capa 3: presenta una resistividad entre 1 $\Omega$ m y $5,4 \Omega \mathrm{m}$. Se correlaciona con la presencia de desechos saturados con lixiviados, caracterizado por una elevada concentración de sales disueltas que favorecen el flujo de la corriente eléctrica; de ahí los valores bajos de resistividad obtenidos. Su espesor varía entre $8 \mathrm{~m}$ y $50 \mathrm{~m}$, alcanzando su profundidad máxima en el SEV5.

- Capa 4: presenta un rango de resistividad entre $49 \Omega \mathrm{m}$ y $360 \Omega \mathrm{m}$. Corresponde con el basamento sobre el cual se depositaron los desechos. Está conformado por lavas de composición andesítica o dacítica, cuyo techo se ubica a partir de los $17 \mathrm{~m}$ y hasta $50 \mathrm{~m}$ profundidad.

Con los ensayos realizados se obtuvo información a una profundidad que ronda los $65 \mathrm{~m}$, con tal grado de detalle que permitió establecer el espesor del depósito de desechos, ubicación de las capas posiblemente saturadas con lixiviado y su distribución lateral. En promedio se tienen 25 $\mathrm{m}$ de espesor de desechos. Además de una aproximación de la morfología del techo del basamento.

Se tiene entonces que el RSRA requeriría la instalación de un sistema de drenaje profundo, el cual permita drenar la (s) capa (s) donde posiblemente se esté dando la acumulación de lixiviados.

Esto no solo sería necesario con el fin de captar dichos lixiviados para que puedan recibir el tratamiento respectivo, sino también porque se estaría evitando que parte de los mismos se infiltren hacia el basamento para luego fluir gradiente abajo y fuera del lindero del relleno sanitario de Río Azul, hacia los acuíferos bajo las poblaciones de San Antonio de Desamparados. Esta situación se considera como una fuente potencial de contaminación a las aguas subterráneas al cambiar la composición química del agua que se extrae para diversos usos.

\section{REFERENCIAS}

ARIAS, M.E., 2002: Aplicaciones de la geofísica a la hidrogeología en Costa Rica.- Rev. Geol. Amér. Central, 27: 11 - 20. 
ARIAS, M.E., 2007: Modelización directa y comparación de los principales dispositivos Geoeléctricos.- Ciencia y Tecnología, 25 (1 y 2): 7 - 23 .

BELMONTE, S.I., JIMÉNEZ, M.E. \& BORTOLOTTI, A., 2008: Estudio de una pluma contaminante producida por lixiviados, utilizando métodos geoeléctricos.Geos, 28(2): 142.

CANTOS, J., 1987: Tratado de geofísica aplicada [ $3^{\mathrm{a}}$ ed.].- 535 págs. Inst. Geol. y Minero de España, Madrid

DENYER, P. \& ARIAS, O., 1990: Geología de la hoja Abra.- Mapa escala 1:50.000. Inst. Geográfico Nac., San José

DENYER, P. \& ARIAS, O., 1991: Estratigrafía de la región central de Costa Rica.- Rev. Geol. Amér. Central, 12: 1 - 59.

FRANCO, J., 1977: Geología de la zona norte de Quebrada Honda, Patarrá, San José, Costa Rica.- 32 págs. Univ. de Costa Rica, San José [Inf. Campaña Geol.]

MEJU, A.M., 2000: Geoelectrical investigation of old/abandoned, covered landfill sites in urban areas: model development with a genetic diagnosis approach.- J. Applied Geophys. 44(2-3): 115 - 150.
MOTA, R., F.A. MONTEIRO, F.A., MATEUS, A., MARQUES, F.O., GONÇALVES, M.A., FIGUEIRAS, J. \& AMARAL, H., 2004: Granite fracturing and incipient pollution beneath a recent landfill facility as detected by geoelectrical surveys.- J. Applied Geophys. 57(1): $11-22$.

ORELLANA, E., 1972: Prospección geoeléctrica en corriente continua.- 523 págs. Paraninfo, Madrid.

POMPOSIELLO, C., 2009: Tomografías eléctricas en el basurero municipal ciudad de Gualeguychú, Provincia de Entre Ríos: evidencias de contaminación.- Rev. Asoc. Geol. Argentina, 64(4): 603 - 614.

ROJAS, I., 2007: Diagnóstico de la generación, recolección y tratamiento de los lixiviados del Relleno Sanitario de Río Azul y propuestas de alternativas tecnológicas para su mejoramiento.- 226 págs. Univ. de Costa Rica, San José [Tesis Lic.]

SUÁREZ, A. \& MALAVASSI, E., 1953: Levantamiento geológico de $\mathrm{La}$ Carpintera.- 57 págs. Univ. de Costa Rica, San José [Tesis Lic.]. 
